\title{
Anthropological Information About a Message Variability
}

\author{
George Losik, Igor Boyko, Vadim Tkachenko, Boris Potapov, Yury Vilchuk
}

United Institute of Informatics Problems of Academy of Science of Belarus, Minsk, Belarus

\author{
Email address: \\ georgelosik@yahoo.com (G. Losik)
}

\section{To cite this article:}

George Losik, Igor Boyko, Vadim Tkachenko, Boris Potapov, Yury Vilchuk. Anthropological Information About a Message Variability. Mathematics and Computer Science. Vol. 5, No. 1, 2020, pp. 1-9. doi: 10.11648/j.mcs.20200501.11

Received: December 6, 2019; Accepted: December 30, 2019; Published: January 30, 2020

\begin{abstract}
The article represents a particular case in the field of the theory of a message encoding and decoding when the message is transmitted from a transmitter to a receiver. In this case human plays a role of the transmitter and receiver. From the positions of the theory of coding and the theory of pattern recognition, considers the phenomenon of anthropological similarity in the anatomical structure of the human sensory-motor system. There is illustration of the importance of the topological similarity of the transmitter and receiver as a source of additional information on the example of an anthropological (species) similarity of the auditory and articulation systems. The results of experiments support the idea the receiver receives additional information or anthropological information about the message, which is decoded not from the message, but from a physical structure of the receiver. Such a transfer is possible with reference to information about human means, articulations, facial expressions and pantomimic poses. It is concluded, in order to convey the knowledge, the physical structure of the information carrier about this knowledge must be the same for the transmitter of the message and the receiver; in other cases, genesis of this additional information a priori impossible.
\end{abstract}

Keywords: Information, Coding, Message Transmitter, Message Receiver, Anthropological Similarity

\section{Introduction}

At the intersection of different sciences, frequently were given answers to questions that scientists of one field could not solve, and the search for solutions turned into a way "to pound water in a mortar". In the field of computer science, A. Y. Friedland [1] and K. K. Colin [2] shows a critical situation in the further study of "pure" information, a channel for its transmission, calculation of data volumes without calculating the "content" of the information message, and the correctness of the concepts of objective and subjective meanings of the message is questioned. In the field of psychology, V. V. Allakhverdov [3] outlines the critical situation in the further study of the psychology of consciousness and states the question of the cause of genesis of consciousness in phylogeny and its role as a cognitive and adaptive tool in human life. To overcome the critical situation, A. Y. Friedland [1] proposes a hypothesis about two components of information and gives to the concept a new definition. The essence of the hypothesis is in an assumption that the information message contains two components (information has two components): data and meaning were the second declared as new and not classic. The consciousness of a person assigns the meaning to the message [16]. The consciousness is a generator of the message meaning created by the person and a detector of the message meaning received by the person. In the context of this hypothesis, the actual question arises: How the meaning is encoded in the brain? To answer this question, the article, from the positions of the theory of coding and the theory of pattern recognition, considers the phenomenon of anthropological similarity in the anatomical structure of the human sensory-motor system.

\section{The Concepts of the Message Transmitter and Receiver}

In most cases, the message is transmitted from the person to person, from the transmitter to the receiver in a discrete code (for example, words) and for the receiver it is sufficient to know the decoding rule (decoding algorithm) of the message. It is enough for a person or computer to know information about a language of the message, the meaning of words, and the decoding algorithm and not necessarily be physically identical to the transmitter [4]. However, a special 
case is possible, for example for two persons, when the physical structure of the transmitter and receiver, as information carriers, is identical. In this case, there is a possibility of genesis of additional information about the received message. The essence of that is in following: if the receiver has a similar structure with the transmitter, then the receiver has the opportunity to learn additional information about the message variability. This particular information about the message variability the receiver can learn not from the message source, but from own physical structure. Strictly speaking, additional information is not about the message itself, but only about the laws of its variability during multiple sending by the transmitter $[14 ; 15]$. At the beginning, in the receiver initial state, information about the message variability not presented. For additional information genesis, a special experiment is needed in the receiver itself in the form of a special sequence of distortion of the message previously accepted as an etalon. Why is it possible? Because, in the case of identity, the variants of the distortion in the receiver completely repeat the variants of the physical variability in the transmitter.

Therefore, firstly the receiver receiving the etalon can skip this experiment or can use it. Skipping the experiment, the receiver collects statistics about the etalon of the recognized message and its range of variability; however, the receiver collects it analyzing only the messages coming from the transmitter. Using the experiment, the receiver receives information from the transmitter only about the etalon message. Zones of its variability are refused for recognition. In the receiver, a sensory etalon of the received message is formed. This is the initial and the first phase of the experiment to reveal additional information. Then, in the second phase, the receiver learns to copy the message, the etalon of which was memorized. For that, the receiver should have a system of imitation and copying the messages similar to those transmitted with the transmitter and which the receiver has learned to recognize. After this, the third phase follows, when the receiver simulates all possible variations of the original etalon. The receiver simulates variations to the reproduced signals by synthesizing such variants of the etalon potentially possible in the receiver and hence the transmitter. These variations are implemented in the motor, rather than in the sensor coordinate system of the etalon. Such an experiment in simulating inaccuracies, according to the hypothesis, the person does only once. At this time, the receiver does not accept external signals; the sensor system receives the variants of the etalon synthesized in the motoric system and enriches the sensory etalons with additional information.

In a result of the experiment, the receiver, using the vector code, adds to the sensor etalon the information about its potential variability $[12 ; 13]$. Additional information about the transmitter appears in the receiver not from the transmitter itself, but transmitted to the receiver as if by a genome, which preserves the species specificity of the organism.

Later, the usefulness of additional information for improvement the reliability of recognition of form-variable objects will be considered in great details. Now, let us illustrate the importance of the topological similarity of the transmitter and receiver as a source of additional information on the example of an anthropological (species) similarity of the auditory and articulation systems of all people.

\section{Oral Speech and Anthropological Information}

Let us consider a usefulness of the topological similarity of the structure of the transmitter and receiver on the example of the child's acquisition of oral speech [5-7]. Studies of children's speech [8] showed that the child learns the phonetic etalon of oral speech syllables (ba, va, gu, bo) in four stages, forming the etalon of the syllable sounding and duplicating it memorization in two places (two indicative spaces).

At the first stage, the child receives information about the syllable sounding in the structure of the word, perceiving its acoustic signal pronounced by an adult. The child learns information about the word sounding; however, cannot recognize information about the permissible inaccuracies of the syllable sounding in the word. The motoric program of word articulation, stored in the adult memory, is not accessible to the child for influencing on it in order to know its variability. Therefore, perceiving the adult speech, the child does not have the opportunity to hear, for example, two variants the same word sounding with some difference in the articulation program. However, a child not only can acquire the oral speech but also reproduce it. For that, he has an articulation apparatus, anthropomorphic in the structure for the child and adult. At the age of 8 to 11 months, the child does not possess the skills of the whole word articulating and does not know how to use articulating movements for the word phonation even though his "babbling" speech already developed. Nevertheless, at this age the child is already able to recognize simple words by the ear.

At the second stage, the child learns how to pronounce words and repeat them and it gradually forms his motor program of the word pronunciation [6]. Moreover, the structure of the motor program of the same speech unit for the child and adult is the same because of the anatomical and physiological isomorphism of the structure of their articulation apparatus. Therefore, the child to learn articulation inaccuracies in the adult pronunciation can repeat it himself, reproducing inaccuracies in his own program for the same speech unit.

At the third stage of word acquiring [8] the child, in turn, performs planned elementary deviations in different parts of the program. At the same time, he always perceives by the ear a pair of realization of a word one after another spoken by himself.

At the fourth stage, "subtracting" the first realization from the second, the child receives and stores in the sensory system the information about the program transformations occurred in the motor system. 
This research [8] showed, the iterations of "babbling" speech serve to the child as such a particular source from which he acquires information about the patterns of the modification of adult speech signals. "Babbling" iterations like "ba-ba-ba", "va-va-va" are acoustic signals by which the child imitates in his speech reproducing system possible inaccuracies in adult speech. Other words, the iterations of child "babbling" are a kind of perceptual action that the human auditory system uses to study the instability of the speech motor system.

For this unique kind of perceptual actions, the subject of perception in addition to the sensory system must have a system for synthesis of the signals he perceives. According to this principle for auditory perception, a figurative perception of the person's facial expressions and pantomime of surrounding people, communicative and expressive gestures of the other person's hand is formed. Any imitation performed by one person (student) towards another (teacher), according to the proposed model, is necessarily accompanied by formation of the student's ability not only to imitate the phenomenon, but also to simulate its inaccuracies in order to be able to perceive it in future.

Such conditions of anthropomorphism are necessary only for transferring into the etalon image of the perceived object the additional information about the instability of its form, but not the form itself. A minimal image of a new object can be formed without this information. In the latter case, the perception of the object also becomes possible, but it will be less reliable, since it will be distorted in the case when the distorted in form object enters to the analyzer. The recognition of the object will be implemented by its invariant characteristics. Such properties will not be supplemented by variation characteristics and the variation patterns will be unused.

So, due to the isomorphism of the structure of the articulation system of the information transmitter (adult) and receiver (child), the receiver can skip taking from the transmitter information about the permissible variations of the etalon. The child learns this additional component of the necessary information about the etalon from his own articulation system.

Let us consider mathematically the four-phase process of decoding the information about the transmitter, which sent by the transmitter in a veiled way because of the identical structure with the receiver. The example illustrates the process of the child's acquisition of oral speech in ontogenesis. The child acquires information not from the adult and not from the outside, but from generated in his sensor-motor system because of the triggering of a special innate mechanism.

\section{Adding Anthropological Information with Mathematical Graph}

In the beginning, with a set of points depict a set of sensory stimuli the child acquires listening and watching the adult. For example, there is the set of syllables $b a, b u$, $b o$ of the child's first words heard from the surrounding (Figure 1a). The set of stimuli could equally be, for example, a set of gestures of an adult's hand, or a set of emotional expressions of the adult face that the child perceives visually.
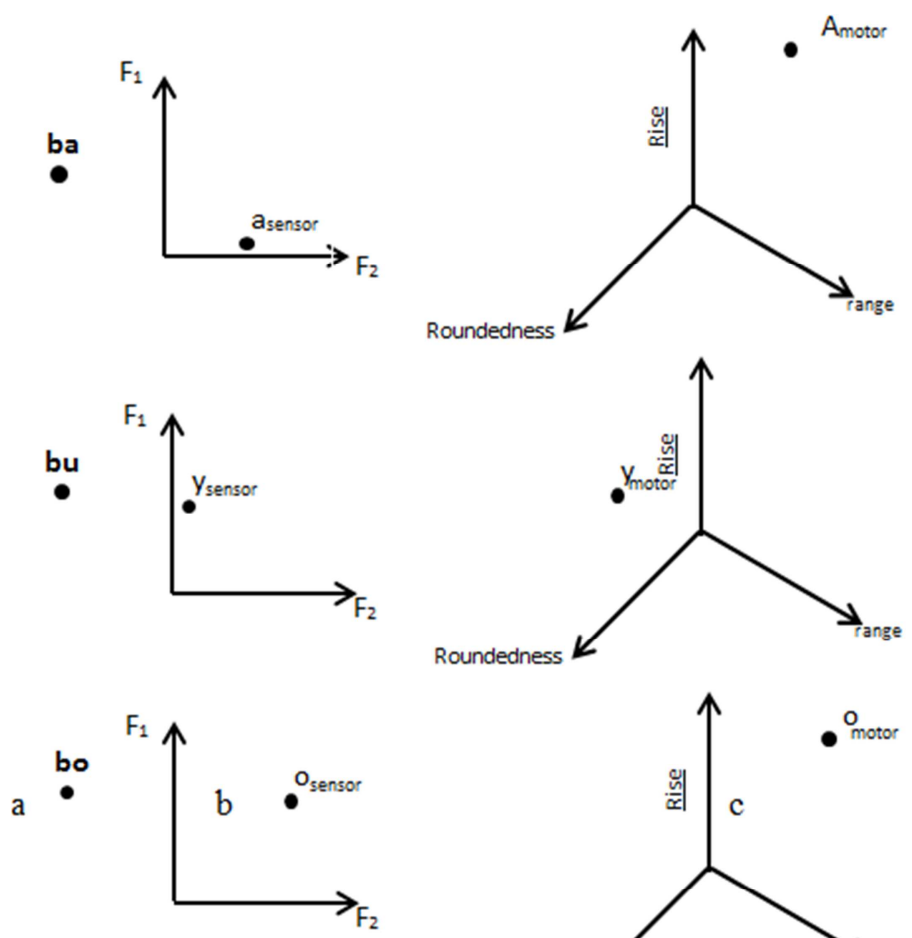

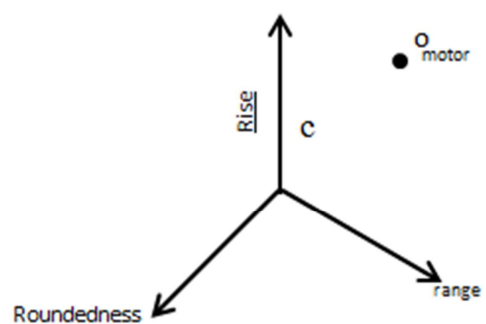

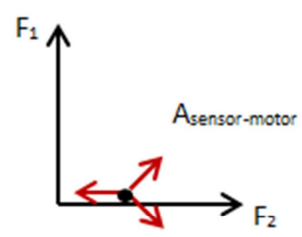
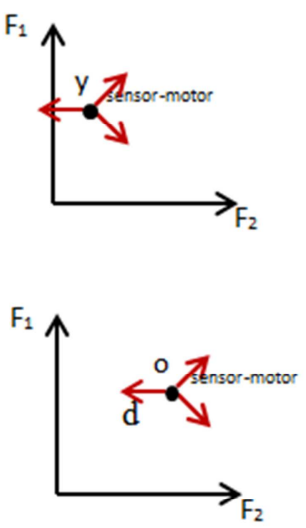

Figure 1. $(a, b, c, d)$. The images by points (a) in the coordinate space of the sensory analyzer (b) and the motor analyzer (c) of the three phonemes a, $u$, when forming their images in the ontogenesis of the child's speech development (d). 
Next, repeat the image of these stimuli with the same points, but in the space of the sensor scales (Figure 1b), in the coordinates of which the acoustic average statistical etalons were formed in the child's auditory system, for example, three syllables $b a, b u, b o$. It is known from phonetics that the vowels $a, u, o$ differ by the first F1 and the second F2 frequency formants. Therefore, in the auditory space of the child who does not know yet how to pronounce them, but knows how to distinguish them by the ear, these three vowels with their etalons will be reflected in the system of values of only two characteristics (Figure 1b). If the child at this level of development hears syllables with these vowels, but with deviations in their frequency spectrum, then he recognizes them only to the extent of the acoustic proximity of the stimulus heard and memorized etalon. This is the first stage in the speech development.

On the next stage of speech development, the child learns the pronunciation of the syllables. From phonetics, it is known that the child, already having sensory etalons, imitates it and places, mastering the pronunciation of these sounds, their motor etalon in the system of articulation properties. There are three articulation properties for vowels: a series, an ascent, a vowel eddiness. Figure $1 \mathrm{~b}$ illustrates it in a new three-dimensional space. The phoneticians have proved that the metric of the acoustic vowel distance (the vowel triangle) differs significantly from the metric of their articulation distance [9]. After that, the third veiled informational stage begins. A child at the age of 5-12 months demonstrates in speech a very specific phenomenon - "babbling", which in the essence is multiple iterations of the same syllable [8]. In the iteration of syllables $b a-b a-b a, b o-b o-b o, b u-b u-b u$ the child models his own articulation system without information about the adult articulation inaccuracy. At the same time, the child listens attentively own babbling speech. Due to this, 3 vectors of three possible directions of articulation distortion of the acoustic reference are added to the acoustic etalon of the two-dimensional sensory space (Figure 1d).

The system of graphs depicts possible variations of the pronunciation of these vowels in the adult speech and possible variants of their distortion. The child, in the future, listening to the adult, has to restore such distortion in the etalon. The graph in Figure 2 depicts transition of the acoustic etalon point of the vowel phoneme to the distorted acoustic point. For example, the child hears a chain of phonemes forming the word "BULONKA", which the adult pronounces with the typical variation, repeating this word many times.

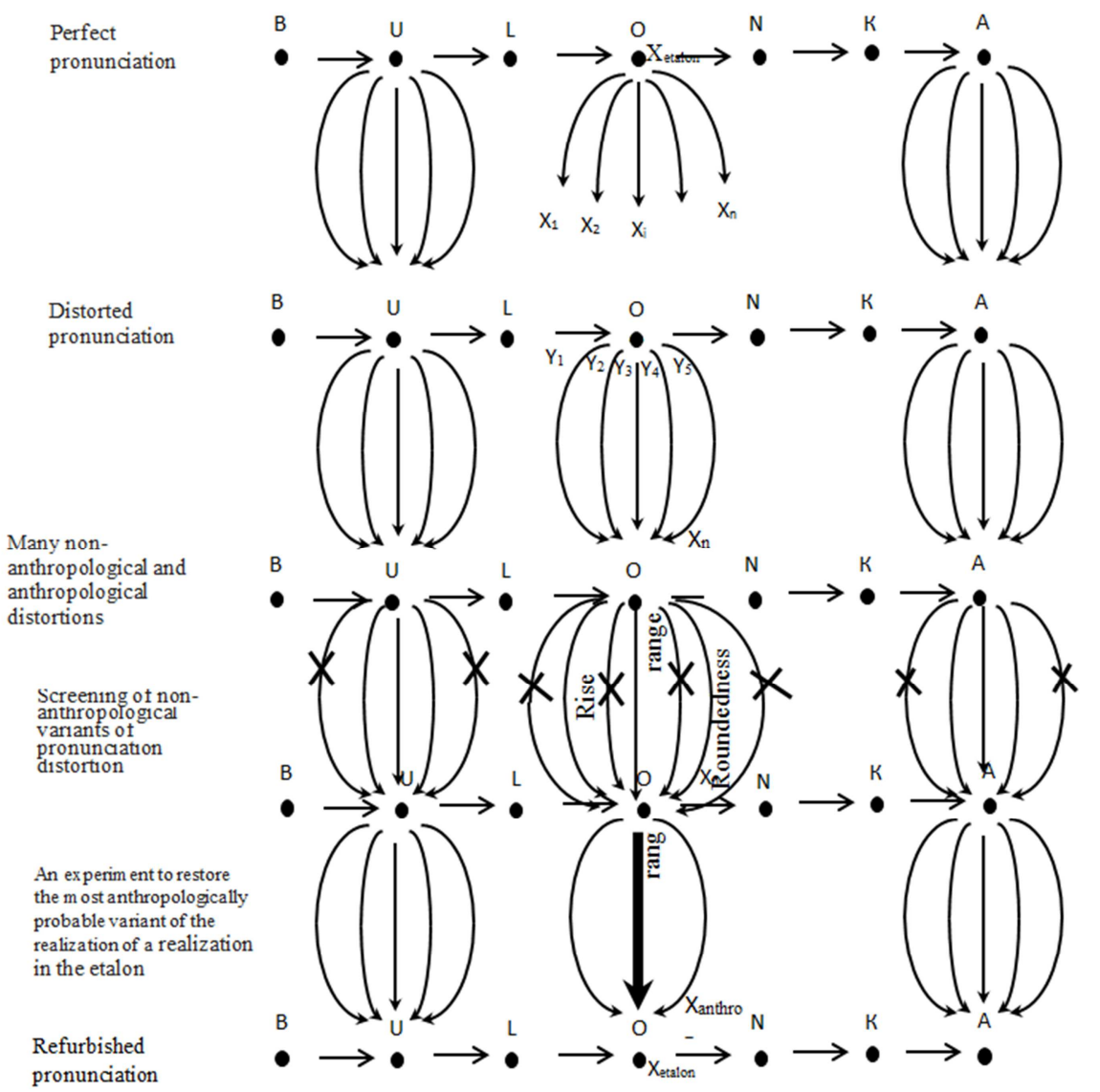

Figure 2. The graph of transition, on the first stage, of the etalon implementation of the vowel phonemes $U$, O, A into acoustically distorted; filtering, on the second stage, of anthropologically unrealistic acoustic implementations; transition, on the third stage, the distorted implementations again into the etalon.

Denote the chain of phonemes $B, U, L, O, N, K, A$ as the chain of random events, and one of these events is the event of pronunciation of the phoneme " $O$ " - will be a random variable $\mathrm{X}$. This event is repetitive, but not identical every time and have articulation transformations. Therefore, the repetition of the phoneme " $O$ " is a set of values of the 
random variable $\mathrm{X}: \mathrm{X} 1, \mathrm{X} 2, \ldots \mathrm{Xi}, \ldots \mathrm{Xn}$. Each of the events $B$, $U, L, O, N, K, A$, like the event " $O$ ", can be considered as some random event $\mathrm{Xj}$, which can be distorted from its etalon $\mathrm{Xj}$ and therefore generate a set of values close to $\mathrm{Xj}: \mathrm{Xj} 1$, $\mathrm{Xj} 2, \mathrm{Xj} 3, \ldots$. At the same time, potentially there are several variants of distortion, for example for vowels $U, O, A$, but unknown yet. This set of distortion can be represented as a set of arcs of the graph (Figure 2, the first stage). The probability of each of them can be represented as the value of some second random variable Y.

As a result, the set of distortions of the vowel in the receiver's input is represented by points. The vowel distorted variants are captured purely by points in the sensor space of the receiver. If the receiver does not repeat the anthropological structure of the transmitter, does not "know" the distortions in the transmitter, and does not know its degree of freedom, then the number of the arcs of the graph belonging to the set $\mathrm{Y}$ and the number of possible variants of transition $\mathrm{X}$ as the point to a distorted state $\mathrm{X}$ as the different point is quite numerous. This is the set of values of the second random variable $\mathrm{Y}$.

Now adding information about the set $\mathrm{Y}$ it is valid to declare that the material source and the cause of the distortions is not any factor, but a purely articulation apparatus for the pronunciation of words and phrases. Anthropomorphism or similarity of the articulation systems of the child (receiver) and the adult (transmitter) dramatically reduces the number of options of the possible transition of $\mathrm{X}$ etalon to $\mathrm{X}$ distortion. From the set $\mathrm{Y}$ of sensory possible arcs of the graph, only the "anthropologically" possible arcs (Figure 2, the second stage) can be left as motoric possible. This additional information essentially makes it easier for the child, when perceiving an unknown phoneme, adaptation the distorted stimulus to the etalon. Such a decrease in uncertainty, which is important, the child is able to do himself (Figure 2, the third stage) only on the basis of the structure similarity of his speech apparatus with the speech apparatus of the adult.

Probably, it explains the fact that repeated long experiments on training monkeys to understand by ear 300400 words have failed. The experiments showed that for the monkey recognition of a relatively large number of words in human speech is not possible. This is consistent with the fact that the monkey articulatory apparatus is fundamentally different from the human articulation apparatus. Also, the experiments showed that recognition the gestures of a human hand is possible for the monkey. Accordingly, it is known that the human and monkey wrists are very similar in anatomy and in the neural control mechanism.

The phenomenon, when for speech perception it is necessary to provide speech articulation, is known as a phenomenon underlying the motor theory of speech perception [10]. However, the theory does not address in details the informational essence of where the increase in recognition reliability comes from when to the motor description is added to the sensory description of the phonetic unit.

\section{Adding Anthropological Information in Terms of the Probability Theory}

As the example, the mechanism of adding anthropological information to the receiver, the mechanism described in terms of the graph theory was considered. The same mechanism may be described in terms of the probability theory. Another mathematical interpretation illustrates the mechanism of the veiled process of adding the anthropological information about the transmitter to the receiver $[11,12]$.

The classic case represents when the anthropological information about the transmitter is not taken into account by the receiver and when it is not transmitted to the receiver in advance. In this case, the maximum amount of information in the chain of implementations Xi1, Xi2,... Xin of the $\alpha$ message received by the sensory system of the receiver from the transmitter will be equal to:

$$
I_{\max }(\alpha)=\sum_{X \in X_{\mathrm{yu}}} P(\alpha) \cdot P(X / \alpha) \cdot \log [P(\alpha) \cdot P(X / \alpha)] .
$$

Taking into account the isomorphism of the structure of the human transmitter and receiver sensor-motor systems it is valid to introduce, in addition to the random variable $\mathrm{X}$, the second random variable Y. After this, the expression for computing the maximum amount of information takes a different form. At the current moment, a portion of the information received from the transmitter in the form of a chain of realizations Xi1, Xi2,... Xin of the message $\alpha$ will be supplemented with another portion. After elimination of nonanthropomorphic hypotheses, the first piece of information will be supplemented with the second piece from the chain of realizations of the variable $\mathrm{Y}: \mathrm{Y} 1, \mathrm{Y} 2, \mathrm{Y} 3, \ldots \mathrm{Yi}, \ldots \mathrm{Ym}$. Therefore, the maximum amount of information in the chain of realizations Xi1, Xi2,... Xin of the message areceived by receiver's sensor system from the transmitter will be equal to

$$
\begin{gathered}
I_{\max }(\alpha)=\sum_{X \in X_{y 4}} P(\alpha) \cdot P(X / \alpha) \cdot \log [P(\alpha) \cdot P(X / \alpha)]+ \\
\quad+\sum_{Y \in Y_{\mathrm{y}}} P(\alpha) \cdot P(Y / \alpha) \cdot \log [P(\alpha) \cdot P(Y / \alpha)] .
\end{gathered}
$$

Thus, the example of adding of unrecorded anthropological information during the child's acquisition of vowel phonemes in his oral-speech communication with the adult is considered. It can be concluded that for the appearance in the receiver of additional information about the transmitter without its participation strict conditions are necessary. At the receiving end, it is not enough of simple physical imitation and not enough of imitation on the level of acoustic, optical, ballistic similarity of the transmitter and receiver signals. When imitating a receiver with the discrete symbols of the transmitter language, for example, the phoneme, grapheme, gesture, posture it is insufficient to have only the physical signal of the phoneme, grapheme, gesture, 
and posture. It is necessary to imitate the physical structure of the transmitter and receiver. Only in this case information about the diversity of the degree of freedom of the sign and its ability to be realized inaccurately can be transmitted by the anthropological similarity of the structure of the transmitter and the receiver of this symbolic information.

\section{Usefulness of Information About the Variability of an Object for the Recognition Reliability}

A human perceiving system accumulates information about the etalons of external phenomena for their recognition. Therefore, there is a question: how is the additional information used after its appearance in the sensor system? Its application, in particular, is possible for increasing the reliability of recognition new variations of previously recognized objects and the constancy of distortion recognition. To isolate the case of anthropomorphic structure of the transmitter and receiver, it is necessary to oppose it to more general case, when the receiver deals with recognition of the form variable object, but not an anthropogenic phenomenon, not speech, gesture, or posture, that the transmitter can imitate. For example, trees, flowers, fish trunks, four-legged animals, birds, ball, umbrella are such form variable objects. The paper [11] completely devoted proving that in general, active perceptual influences of a person on this object are necessary for decoding the laws of variation of the object. Only under artificial, human-induced variability, the perceiving system can perform a "decomposition" of the object variations to a small set of vectors of elementary variations. If the object is significantly variable in the form the perceptual actions are needed because the study of only zones of variation is less effective. However only in the special case, if the receiver can copy the perceived object it can perform perceptual actions not with the object itself, but with its copy.

First consider a more general case, when the object is variable, perceptual actions are performed with it, and its recognition is carried out taking into account information on its variability. Strictly speaking, the session of the sensory contact of the subject with the external object transmits to the subject only a chain of photographs. But from the point of view of semantic work of the brain, it is simultaneously a chain of messages about the motive of the perceiving subject from the point of usefulness for him to recognize the object of perception. In the chain of input raw data for the forthcoming sensory decoding, there is a lot of uncertainty about in which way (from the set of sensory possible ways of transformation) the given perceived object deviated from the etalon. There is an uncertainty about the possible degree of its deviation freedom and the uncertainty of the object choice of the transition way in the transformation of its form. This is due to the fact that such raw data are not generalized by knowledge of the material structure of the transmitter, which generates inaccuracies. According to the proposed hypothesis, the sensory system of the child and the appeared here sensory etalon receives information about the anthropological experiential system of the adult. This information reduces the possible number of alternatives.

\section{Non-classic Algorithm for Recognizing the Variable Form Object}

The article [11] shows the necessity of three times study of the object to determine probabilistic characteristics $P(X / \alpha)$, $P(Y / \alpha), P(\alpha)$, and in each of the cases the object is studied under different conditions of existence. The order of the study $P(X / \alpha)$ and $P(Y / \alpha)$ may be arbitrary, while the search for $P(\alpha)$ without a teacher is possible only after finding $P(X /$ $\alpha)$ and $P(Y / \alpha)$.

Let us assume that the system sequentially through the three specified stages learned to recognize a number of objects $\alpha_{1}, \alpha_{2}, \ldots, \alpha_{k}, \ldots, \alpha_{n}$. Then during recognition, when the implementation of $X$ of an unknown object comes at its input, according to the implementation and information about $\mathrm{P}(X /$ $\left.\alpha_{k}\right)$ and $\mathrm{P}\left(Y / \alpha_{k}\right)$ of each of the objects $\alpha_{k}(k=1,2, \ldots, n)$ the system computes $n$ hypotheses about the belonging of $X$ to each of the $n$ objects. In correspondence with the most reliable hypothesis, a decision is made regarding the input object $\alpha_{i}$ entrance.

The correctness of this recognition problem not decreases if instead of the group of objects just one object $\alpha$ will be considered, and its recognition will be in opposition to the object "not $\alpha . "$ If the hypothesis about the object $\alpha$ is not confirmed $P(\alpha / X)<0.5$, then it is confirmed that with the probability $(1-P)$ the contrary hypothesis is in favor for the object "not $\alpha$ ", and no other parallel hypotheses about other objects. Therefore, instead of computing $n$ possible hypotheses let us consider the algorithm for computing the hypothesis about belonging the input implementation of $\mathrm{X}$ to only one object $\alpha$ (or the object "not $\alpha$ ").

\section{The Decision Making Algorithm for the Variable Object}

Consider an algorithm for deciding whether to recognize an object in the case that it is highly variable in its form and if, therefore, it is required to obtain additional information about its variability and use it. We will denote the probability distribution of the form of the studied object $\alpha$ by $\mathrm{P}\left(X / \alpha^{0}\right)$ and the average statistical value of its form is called the standard of the object $\alpha$ and denoted by $\alpha^{0}$. It was noted above that if an unknown new realization $\mathrm{X}$ of the recognizable object arose due to an insignificant transformation of its form relative to $\alpha^{0}$, then the similarity of $\mathrm{X}$ when it is compared with $\alpha^{0}$ is easy to find. In this case, it is possible to decide whether $\mathrm{X}$ belongs to the object $\alpha$ without resorting to information about the laws of its variation. To test the hypothesis that $\mathrm{X}$ belongs to the object $\alpha$ it suffices to use the well-known Bayes formula of the following form [13]: 


$$
P\left(\alpha_{i} / X\right)=P\left(\alpha_{i}\right) \cdot P\left(X / \alpha^{i}\right) / \sum P\left(\alpha_{k}\right) \cdot P\left(X / \alpha^{k}\right) .
$$

At the same time, it may turn out that the input implementation does not show any similarity with the object's standard $\alpha$ and the value of $P(\alpha / X)$ is close to 0.5 . Therefore, neither the hypothesis of belonging $X$ to $\alpha$, nor the hypothesis that $\mathrm{X}$ belongs to "not $\alpha$ " is confirmed. The reason for this can be a strong dissimilarity in the realization of $\mathrm{X}$ with the standard $\alpha^{0}$ due to a significant transformation of the shape of the object $\alpha$. In this case, the information accumulated in the analyzer about the "grammar" and "lexicon" of anthropologically possible transformations of the object $\alpha$ becomes useful. Information on the variability of the object $\alpha^{0}$ and its parts $\alpha$ are presented in the distributions $P\left(Y / \alpha^{0}\right)$ and $P(Y / \alpha)$, where $\psi=1,2, \ldots$. In this case, information about the laws of variance can be used to increase the similarity between the implementation of $\mathrm{X}$ and the standard of the object whose transformation it represents.

\section{The Need for an Experiment to Fit the Stimulus to the Benchmark}

There are two ways to use information about variability when comparing the input implementation and the reference. On the first, the system can try to bring them closer by fitting the standard for implementation. In this case, the grammar of transformations with the object that was detected earlier and stored in the receiver is performed on the object in the internal plane, i.e., internalized: this grammar transforms the stored standard of the object. Reproduction in the receiver in the internalized plan is not the transformations themselves, but their grammar makes the receiver capable of extrapolating all theoretically possible states of the object, which, if not observed in the study of the object, may occur after training.

The case of fitting the standard for implementation is applied when the receiver cannot create a copy of the recognized phenomenon when it is not anthropomorphic in structure to the transmitter. At the same time, the same effect of the rapprochement of the implementation with the standard can be achieved by a second fit, not a standard for implementation, but implementation of the standard. To do this, knowing the grammar of the object's transformation outside, the analyzer performs an inverse operation: makes attempts to restore the initial reference implementation $\alpha^{0}$ from the transformed implementation $\mathrm{X}$. To this end, previous transformations are performed on the input realization and its parts according to grammatical rules, but not in the direct, but in the opposite direction. Internalized transformations in the normalization of the input signal are carried out by trial and error in turns in different directions, until either of them leads to an increase in the similarity between the normalized implementation and the standard of one of the known objects.

Consider the decision algorithm for recognizing the input implementation using information about the variability of the perceived object. We will proceed from the assumption that, at the stage of studying the object $\alpha$ according to the work, the anthropological information on the variability of the message the receiver received:

i. statistical information on the instability of its form (the probability distribution $\mathrm{P}(\mathrm{X} / \alpha)$ ) and the shape of its parts to the level $\alpha$ inclusive (probability distributions $\mathrm{P}(\mathrm{X} / \alpha)$ ), from which the average statistical standards $\alpha$ ${ }^{0}$ of the shape of the object and its parts $\alpha$;

ii. information on the directions of transformation of its form (the distribution of $\mathrm{P}(\mathrm{Y} / \alpha)$ ) and its parts (the distribution of $\mathrm{P}(\mathrm{Y} / \alpha))$, from which the mean statistical values of these directions Y0, Yare found;

iii. information about the frequency $\mathrm{P}(\alpha)$ of the appearance of the object $\alpha$.

If you do not change the standard when entering the implementation of $\mathrm{X}$ on the input, that is, do not customize the reference to the implementation and calculate the degree of its similarity to the standard by the formula (1), then the information on the path $\mathrm{Y}$ of the transition of the object to state $\mathrm{X}$ will remain un-decoded. Therefore, in order to implement the $\mathrm{X}$, decode variable $\mathrm{Y}$, to find out its value, the receiver must arrange with the input and the standard as input a kind of experiment on fitting them to each other.

Suppose that the option is selected, at which the standard is adjusted for implementation. The adjustment is made by the same variable $\mathrm{Y}$, which is responsible for the transformation of the standard $\alpha^{0}$ into the implementation of $\mathrm{X}$, but works in the opposite direction. The formula for carrying out experimental attempts to find the happened transformation $\alpha^{0}$ in $\mathrm{X}$ is as follows:

$$
X^{\mathrm{U}}=Y_{X} \cdot\left\{\sum Y_{X^{1}}\left[\sum Y_{X^{2}} \cdots\left(\sum Y_{X^{\Psi}}\left(X^{\Psi}\right)\right)\right]\right\},
$$

where $\mathrm{X}$ is the part of the original realization $\mathrm{X}$ of the unknown object formed when the $\psi$ fold decomposition of it (for $\psi=0$, the value of $\mathrm{X}$ corresponds to the whole realization of $\mathrm{X}$ as a whole);

$X^{\mathrm{H}}$ - artificial implementations, formed as a result of experimental attempts to adjust the initial realization of $X$ to the standard $\alpha^{0}$ by transforming it and its parts $\mathrm{X}$;

$Y_{\mathrm{X}}$ - transformations in the direction opposite to the transformations;

$Y_{X^{\Psi}}$ - transformations in the direction inverse to the transformations $\mathrm{Y}$, extending to the part $\mathrm{X}$ of the realization of the $\mathrm{X} \psi$-fold partition.

In formula (2), the known quantities are the value of $X^{n}$, which, as a result of the experiment, should assume a value equal to $\alpha^{0}$ and the value of $\mathrm{X}$, while the quantities $Y_{\mathrm{x}}=-Y_{\alpha^{0}}$, $Y_{X^{\Psi}}=-Y_{\alpha^{\psi}}$ turn out to be unknown, since they can take for one and the same, but the object of one and the same part of it has several values. The experiment in the receiver with respect to fitting $\alpha$ under $\mathrm{X}$ is directed precisely at the solution of formula (2). Mathematically, it is expressed by the fact that by substituting in the formula (2) for different values of $Y_{\mathrm{x}}$ and $Y_{X^{\Psi}}$ in different combinations of them, 
there are values of $Y_{x}^{\max }$ и $Y_{x^{\psi}}^{\max }$, that is, those values that best satisfy the given equation. If the input implementation of $\mathrm{X}$ is an implementation of the object $\alpha$, then in a sequential search of all possible transformations, one will inevitably be found such that from $\mathrm{X}$ form's an artificial realization of $X^{\mathrm{umax}}$, which is close enough to the standard $\alpha^{0}$. It is this transformation of $Y^{\max }$, which in the simulation provides the transformation of $\mathrm{X}$ into a close to the standard realization of $X^{\text {umax }}$, corresponds to the sought value of the random variable Y. If the input implementation is not a realization of the object $\alpha$, then in the artificial realization experiment, close to the standard $\alpha^{0}$, it will not be detected. The receiver after such an experiment has the right to decide that the input implementation belongs to the object "not $\alpha$ " and proceed to a new experiment to fit the implementation of $X$ to the standard of another object known to it, for example $\beta$.

Thus, it is only thanks to the experiment with the arrival of the implementation of $\mathrm{X}$ on the analyzer's input, indirectly by the current value of the change $X$ and the past information about the subject's an anthropological variability stored in memory in the inactive representation in the realization of the second value - Y. As a result, the receiver receives information via $\mathrm{X}$ about one, but about two unknown quantities that characterize both the form and the variability of the perceived object. Before the experiment, the appearance at the input of the description of the implementation of $\mathrm{X}$ removes the uncertainty only about the form of the object. After the experiment and the solution of formula (2), the indeterminacy of the choice of the object for the transition to the state $\mathrm{X}$ is removed.

Information on the values of the quantities $Y_{x}^{\max }$ and $Y_{x^{\psi}}^{\max }$ indicate the path of the inverse transformation of the input realization $X$ and its parts into the standard $\alpha$. Therefore, instead of calculating the hypothesis $P(\alpha / X)$ according to formula (1), it becomes possible to apply the more precise formula (3) to calculate the hypothesis that the implementation of $X$ belongs to the object $\alpha$. Conditionally, we can assume that the information about the identifiable object to the receiver is carried by two events arriving at its input: $\mathrm{X}$ and $\mathrm{Y}$, since the event $\mathrm{X}$ is always accompanied by the event Y. With this in mind, we can write:

$$
\begin{aligned}
& P(\alpha / X) \rightarrow \text { Experiment } \rightarrow P\left(\alpha / X^{\max }, Y^{\max }\right)= \\
& =P(\alpha) \cdot P\left(X^{\text {Иmax }}, Y^{\text {Иmax }} / \alpha\right)=P(\alpha) \cdot P\left(X^{\text {Imax }} / \alpha\right) \cdot P\left(Y^{\text {Umax }} / \alpha\right)
\end{aligned}
$$

The events $\mathrm{X}$ and $\mathrm{Y}$ in their physical nature with respect to the object * can be considered independent. More strictly, before the occurrence of the event $\mathrm{X}$, the event $\mathrm{Y}$ has a large set of possible values found in all the objects studied. With the arrival of a specific event $X$ after the calculation of formula (1), the object * corresponding to the event $\mathrm{X}$ becomes known with a certain probability. Since the event $\mathrm{X}$ with some probability predefines its object * with its value, this means that it determines the set of possible values of the event $\mathrm{Y}$, narrows it down to $Y_{\alpha}$. Therefore, before the adoption of the intermediate solution by formula (1), the event $\mathrm{Y}$ can be regarded as dependent on the event $\mathrm{X}$. However, after the intermediate solution, at the stage of further refinement with the help of formula (3), the events $X$ and $Y_{\alpha}$ appear as independent, since the value of $\mathrm{X}$ does not influence to the value of $Y_{\alpha}$.

Thus, it is essential that in the formula (3), not the initial value of the input quantity $\mathrm{X}$ is taken for calculations, but the artificial realization of $\mathrm{X}^{\mathrm{umax}}$, which is the result of the best fit of the realization of $X$ under the reference standard $\alpha^{0}$. At the same time, the quantity $Y^{\max }$ appears in the calculations along with the value of $X^{\text {umax }}$

Thus, the three kinds of information about the object $\alpha$ : $P(\alpha), P(X / \alpha), P(Y / \alpha)$, allow us to calculate hypotheses about the degree of similarity of unknown implementations of $\mathrm{X}$. New in this calculation is $P(Y / \alpha)$ is that the criterion for evaluating the similarity of $\mathrm{X}$ and $\alpha$ is not represented by the metric of measuring the form $X$ and $\alpha$, but by the measurement metric of the transformability in real life $X \mathrm{~B} \alpha$.

Based on the consideration of this phenomenon from the standpoint of probability theory, according to [11], the following conclusions can be drawn:

1. In the event that the object of perception is highly variable in its form, in relation to the receiver it acts as a generator of not one but two random quantities. The second of these is the kind of transformation that an object chooses when passing from one form state to another. The larger the object's choice of trajectories for the transformation of its shape, the greater uncertainty for the receiver is represented by this dynamic and the more additional information can be obtained if along with the shape of the object, the dynamics of its shape is also studied.

2. A variable that reflects the dynamics of an object can be represented by a vector quantity $Y$. To find one of its values, it is necessary to know two scalar quantities - $X_{t}$ and $X_{t+\Delta t}$, describing the form of the object under study strictly before and after the artificial impact on it.

3. The calculation of the value of $Y$ in the usual educational sample Such, consisting of single realizations, is fundamentally impossible. In reality only an artificial method is possible that allows one to obtain a training sample HUCH consisting of pairs of realizations for the calculation of the value of Y. It is expressed in the transition of the receiver to the mode of active action on the object and synchronous measurement of its states at the moments of the beginning and termination of each new effect.

4. To calculate the degree of similarity between the unknown realization of $\mathrm{X}$ and the object $\alpha$, three types of information are needed: $P(\alpha), P(X / \alpha)$ and $P(Y / \alpha)$. A new kind of information in this calculation is $P(Y / \alpha)$. Using her estimation of the similarity of $\mathrm{X}$ and $\alpha$ is given not only by the metric of similarity of their form, but also by the metric of transformability in real life $X \mathrm{~B} \alpha$.

5. For the formation of a dynamic image (standard) of the studied object, if it has a significant variability, it is 
necessary to have three procedural conditions (phenomena):

i. the receiver must have a mechanism of active influence on the object;

ii. Such impacts should be one-coordinate and transient and cause a jump in the state of the object;

iii. the receiver must have a mechanism for receiving descriptions of the object at the moments before and after exposure to it.

\section{Conclusion}

Along with imitation of activity, we introduce the notion of imitation of a structure. The transfer of the isomorphic structure of the sensor-motor system of the information receiver by the genome from person to person is to repeat the physical structure that the transmitter has. Due to the preservation of the anthropomorphic structure of the sensormotor system in people as a species, it becomes possible to transmit information between them according to the analogous coding principle, and not only algorithmic, sign. Such transfer is possible with reference to information about human means, articulations, facial expressions and pantomimic poses.

In this case, the preservation in the phylogeny of the constancy of the brain structure, the sensory and motor systems are explained not only from the point of view of Darwin's theory of natural selection and the survival of human as a corporeal being, representative of the species, but also "survival" of one of the ways of transmitting information from the individual to the individual. The isomorphism of the material structure of one individual as the transmitter of the message and the second as the receiver in certain cases can serve as a mechanism for encrypting and deciphering information on the spatial-topological properties of the message transmitted from the transmitter to the receiver.

Knowledge received and understood by one group of people, encoded by oral, written, gestural symbols, that is, discrete code, should not cease to exist. They must go from the past to the future and must be decoded by another group of people. We come to the conclusion that in order to convey the knowledge, the physical structure of the information carrier about this knowledge must be the same for the transmitter of the message and the receiver. This serves as another argument, why in the phylogenies of human, the constancy of the structure of the brain, the structure of the body, is preserved.

One can make a far-reaching conclusion about the prospects for the development of systems for automatic pattern recognition and mechanical imitation systems for humans. When imitating a phoneme, grapheme, gesture, posture, as discrete symbols of the transmitter language, it is not enough to imitate at the receiving end at the level of acoustic, optical, ballistic similarity of the phoneme, grapheme, gesture, posture physical signal. It is necessary to imitate the material structure of the transmitter and receiver.
For information on the diversity of degrees of freedom of a sign is not accurately realized - it is transferable only with the anthropological similarity of the structure of the transmitter and the receiver of this symbolic information.

\section{References}

[1] Fridland, A. Ya. On the essence of information: two approaches. Information Technology. 2008. - No. 5. - P. 75-84.

[2] Colin K. K. Criticism of some methodological approaches in informatics and information education. Open Education. 2005, №2. Pp. 81-89.

[3] Allahverdov, V. M. Consciousness in the logic of knowledge. Materials of the Fifth International Conference on Cognitive Naca. 18-24 June 2012, Kaliningrad. C. 216.

[4] Shannon K. Works on the theory of information and cybernetics: Per. with English. / K. Shannon - M.: Foreign Literature, 1963. - $830 \mathrm{p}$.

[5] Beltyukov V. Interaction of analyzers in the process of perception and assimilation of oral speech. - M., Pedagogika, $1977.176 \mathrm{p}$.

[6] Beltyukov V. The program for mastering children by the pronunciation of the sounds of speech. Questions of psychology. - 1979 - No. 4 - P. 66 - 78.

[7] Beltyukov V., Salakhova A. Ya. On the child's mastering of the phonemic (phonemic) language system. Questions of psychology. - 1975 - No. 4 - P. 71-80.

[8] Losik G. Perceptual actions in the perception of speech. Minsk, Institute of Technology. cybernetics NAS of Belarus, 2000. $168 \mathrm{p}$.

[9] Bondarko L. Useful attributes and hierarchical organization of phonemic classification. Sound structure of language. - M., 1979 - P. $20-26$.

[10] Galunov V., Chistovich L. On the connection of the motor theory with the general problem of speech recognition. Acoustic journal, 1965 - Vol. 2. - Issue. 4. - P. 417-426.

[11] Losik G. Perceptual actions of man: the cybernetic aspect, Minsk, 2008, 147 p.

[12] Losik G. Coding information in the brain, LapLambertAcademic Publishing, 2015, 135 p.

[13] Abney D., Dale R., Kello C., Louwerse M. Burstiness across multimodal human interaction reveals differences between verbal and non-verbal communication. CogSci. - 2017. - P. $39-44$

[14] Battleday R., Peterson J., Griffiths T. Modeling human categorization of natural images using deep feature representations. CogSci. - 2017. - P. 111-118.

[15] Halpern D., Gureckis T. Categorization, Information Selection and Stimulus Uncertainty. CogSci. - 2017. - P. 464-470.

[16] Konovalova E., Le Mens G. Selective Information Sampling and the In-Group Heterogeneity Effect. CogSci. - 2017. - P. 688-694. 